\title{
Controllable Kerr Nonlinearity via Incoherent Pump Fields in Semiconductor Quantum Wells
}

\author{
Hamid Reza Hamedi $^{1,2, *}$, Mostafa Sahrai ${ }^{1,2}$ and Habib Tajalli ${ }^{1,2}$ \\ ${ }^{1}$ Photonics Excellence, University of Tabriz, Tabriz, Iran \\ ${ }^{2}$ Research Institute for Applied Physics, University of Tabriz, Tabriz, Iran
}

Received: 2 Jun. 2013, Revised: 18 Jul. 2013, Accepted: 21 Oct. 2013

Published online: 1 Dec. 2013

\begin{abstract}
A theoretical investigation is carried out into the impact of incoherent pumping fields and quantum interference induced by incoherent pumping fields on the Kerr nonlinearity in a three-level asymmetric semiconductor quantum well system. It is found that Kerr nonlinearity is so sensitive to the rate of incoherent pumping fields, but insensitive to the quantum interference induced by incoherent pumping fields. In addition, it is demonstrated that an enhanced Kerr nonlinearity with reduced absorption can be achieved just via the strength of Fano- type interference and without applying any coherent laser field. The obtained results are very different from the conventional schemes which coherent coupling fields are used to control the Kerr nonlinearity. This advantages make our electronic medium much more practical than its atomic counterpart due to its flexible design and the controllable interference strength.
\end{abstract}

Keywords: Kerr nonlinearity, Linear and nonlinear probe absorption, Fano interference, Incoherent pumping field, Quantum interference induced by incoherent pump.

\section{Introduction}

Linear and nonlinear response of an atomic system for the external fields can be modified by quantum coherence and quantum interference. In fact, quantum coherence and quantum interference are the basic mechanisms for modifying the linear and nonlinear absorption and dispersion. There are many interesting phenomena in quantum optics and atomic physics, induced by quantum coherence and quantum interference. Examples include electromagnetically induced transparency (EIT) [1,2,3]], coherent population trapping (CPT) [4], cancelation of spontaneous emission [5], superluminal and subluminal light propagation [6,7], lasing without inversion [8,9], and so on. The effect of electromagnetically induced transparency (EIT) has led to many interesting nonlinear optical phenomena. Two interesting nonlinear optical phenomenon, which has been studied in the recent years in the atomic media are Kerr nonlinearity [10,11,12, $13]$ and optical bistability $[14,15,16,17]$. It is shown that a large Kerr nonlinearity with reduced probe absorption causes the nonlinear optics to be studied at low light power $[18,19]$. In fact, it is desirable to have large third-order susceptibilities under conditions of low light power and high sensitivities, since it can be used for realization of single-photon nonlinear devices. This requires that the linear susceptibilities should be as small as possible for all pump and absorption loss. Giant Kerr nonlinearity may lead to many important applications in nonlinear phenomena, for example; multiple usages of giant Kerr nonlinearity in quantum information process (QIP) enable us to detect and resolve individual optical number states, such as quantum bit regeneration [20], long-distance quantum teleportation [21], Bell-state measurements [22], optical Fock state synthesis [23] and so on. Optical bistability, on the other hand, in multi-level atoms confined in an optical ring cavity has recently been developed due to its potential application in all optical switching and optical transistors which are necessary for quantum computing and quantum communications [24]. Several methods were described to achieve large Kerr nonlinearity with reduced absorption as well as controllable $\mathrm{OB}$ and $\mathrm{OM}$ in an EIT medium [25,26,27, 28]. As an important proposal, Harris and Yamamoto [29] described the creation of an absorptive optical switch in a four-level atomic system due to quantum interference. Experimental realization of such photon switching by quantum interference in a four-level atomic system was described by Yan etal. [30]. In Ref. [31] the effect of laser

\footnotetext{
*Corresponding author e-mail: Hamid.R.Hamedi@gmail.com
} 
intensity and quantum interference induced by spontaneous emission on optical bistability, optical multi-stability as well as Kerr nonlinearity of a four-level Y-type atomic system was discussed. It is found that an enhanced Kerr nonlinearity accompanied with reduced absorption can be achieved. As a result, the controlling parameters can efficiently decrease the linear and nonlinear absorption of the probe field. This may enhance the Kerr nonlinearity of the medium, which makes it easier for the cavity to reach saturation. Thus, a significant decrease of the multi-stable threshold can be achieved. Besides atomic systems, Optical bistability (OB) and Optical multi-stability (OM) as well as Kerr nonlinearity have been studied in other kinds of systems, such as semiconductor structures $[19,32,33,34]$. One of the most interesting solid state medium is semiconductor quantum well (SQW). The reason for this is that achieving large nonlinearities in SQWs have many important applications in solid- state quantum information science and optoelectronics. Quantum wells have more capability to provide high nonlinear optical coefficient and large electric- dipole moments. Recently, we (with collaborators) investigate an enhanced Kerr nonlinearity in a four subbund tunnel-coupled double quantum wells [19]. It is found that the energy splitting of the two upper levels and intensity of coupling field can enhance the Kerr nonlinearity and reduced the linear and nonlinear absorption. However, in this paper, an asymmetric double quantum wells (QWs) driven coherently by a probe laser field is proposed to achieve a giant Kerr nonlinearity with negligible absorption. The presented scheme is based on the Ref. [34], but our scheme is much more practical than its counterpart. First, we could achieve an enhanced Kerr nonlinearity via the strength od Fano-type interference, and without applying any coherent control field, which is completely different from what we discussed in our previous work [19]. Second, the incoherent pumping field is used to control the Kerr nonlinearity. Namely, the underlying mechanism is very different from the conventional schemes.

\section{Models and Equation}

The semiconductor double QW structure consisting of two quantum wells that are separated by a narrow barrier is shown in Fig. $1[35,36]$. The $|a\rangle$ and $|b\rangle$ are the first subband of the shallow well and the second subband of the deep well, respectively, which are resonant (see Fig. $1)$. Due to the strong coherent coupling via the thin barrier, the levels split into a doublet levels $|2\rangle$ and $|3\rangle$ which arise from the mixing of the states $|a\rangle$ and $|b\rangle$, under the exactly resonant conditions, $|2\rangle=(|a\rangle-|b\rangle) / \sqrt{2}$ and $|3\rangle=(|a\rangle+|b\rangle) / \sqrt{2}$. The splitting energy $\omega_{s}$ on resonance is given by the coupling strength and can be controlled by adjusting the height and width of the tunnelling barrier with applied bias voltage [35]. A low intensity pulsed laser field $\omega_{p}$ (amplitude $E_{p}$ ) is applied to the transitions $|1\rangle \rightarrow|3\rangle$ and $|1\rangle \rightarrow|2\rangle$ simultaneously with the respective Rabi frequencies $\Omega_{p 1}=\mu_{31} E_{p} / 2 \hbar$ and $\Omega_{p 2}=\mu_{21} E_{p} / 2 \hbar$, with $\mu_{21}$ and $\mu_{31}$ being the intersubband dipole moments of the respective transition. The small signal absorption of the weak-probe field propagating through such a system can be computed in the steady state.

In the interaction representation and under the rotating-wave approximations, the semi-classical Hamiltonian describing the interaction for the system under study is given by $(\hbar=1)$

$$
H_{\text {int }}=\Delta_{p}(|2\rangle\langle 2|+| 3\rangle\langle 3|)-\Omega_{p}(|2\rangle\langle 1|+| 3\rangle\langle 1|+\text { h.c }) .
$$

Where the probe laser detuning is defined as $\Delta_{p}=\omega_{0}-\omega_{p}$ while the average transition frequency is denoted by $\omega_{0}=$ $\left(\omega_{2}+\omega_{3} / 2\right)$.

By adopting the standard approach [37,38,39], we can easily obtain the density-matrix equations of motion in electro- dipole and rotating-wave approximations as follows:

$$
\begin{array}{r}
d \rho_{12} / d t=-\frac{\gamma_{21}}{2} \rho_{12}+i\left(\Delta_{p}-\frac{\omega_{s}}{2}\right) \rho_{12}+ \\
i \Omega_{p 2}\left(\rho_{22}-\rho_{11}\right)-i \Omega_{p 1} \rho_{32}-\frac{\eta}{2} \rho_{31}, \\
d \rho_{13} / d t=-\frac{\gamma_{31}}{2} \rho_{13}+i\left(\Delta_{p}+\frac{\omega_{s}}{2}\right) \rho_{13}+ \\
i \Omega_{p 1}\left(\rho_{33}-\rho_{11}\right)-i \Omega_{p 2} \rho_{23}-\frac{\eta}{2} \rho_{12}, \\
d \rho_{23} / d t=-\frac{\gamma_{32}}{2} \rho_{23}+i \omega_{s} \rho_{23}+i \Omega_{p 2} \rho_{13}-i \Omega_{p 1} \rho_{21} \\
-1 / 2(\eta-\eta)(\rho 33+\rho 22), \\
d \rho_{22} / d t=-\gamma_{2} \rho_{22}-i \Omega_{p 2}\left(\rho_{12}-\rho_{21}\right) \\
-1 / 2 \eta\left(\rho_{23}+\rho_{32}\right) \\
d \rho_{33} / d t=-\gamma_{3} \rho_{33}-i \Omega_{p 1}\left(\rho_{13}-\rho_{31}\right) \\
-1 / 2 \eta\left(\rho_{23}+\rho_{32}\right) \\
\rho_{11}+\rho_{22}+\rho_{33}=1 .
\end{array}
$$

Here $\omega_{2}=\left(E_{3}-E_{2}\right)$ is the energy splitting between the upper levels, given by the coherent coupling strength of the tunnelling. The population decay rates and dephasing decay rates are added phenomenologically in the above equations [40]. The population decay rates for subband $|i\rangle$ denoted by $\gamma_{i}$, are due primarily to longitudinal optical (LO) phonon emission events at low temperature. The total decay rates $\gamma_{i j}(i)$ are given by $\gamma_{21}=\gamma_{2}+\gamma_{12}^{d p h}, \gamma_{31}=\gamma_{3}+\gamma_{13}^{d p h}$ and $\gamma_{32}=\gamma_{3}+\gamma_{2}+\gamma_{32}^{d p h}$ where $\gamma_{i j}^{d p h}$ determined by electron-electron, interface roughness, and phonon scattering processes, is the dephasing decay rate of the quantum coherence of the $|i\rangle \rightarrow|j\rangle$ transition. The parameter $\eta=\sqrt{\gamma_{2} \gamma_{3}}$ represents cross coupling between the states $|2\rangle$ and $|3\rangle$ via the longitudinal optical (LO) phonon decay, it describes the process in which a phonon is emitted by subband $|2\rangle$ and is recaptured by subband $|3\rangle$. These mutual coupling 
terms can be obtained if tunnelling is present, e.g., through an additional barrier next to the deeper well. As mentioned above, levels $|2\rangle$ and $|3\rangle$ are both the superpositions of the resonant states $|a\rangle$ and $|b\rangle$. Because the subband $|b\rangle$ is strongly coupled to a continuum via a thin barrier, the decay from state $|b\rangle$ to the continuum inevitably results in these two dependent decay pathways: from the excited doublet to the common continuum. That is to say, the two decay pathways are related: the decay from one of the excited doublets can strongly affect the neighbouring transition, resulting in the interference characterized by those mutual coupling terms. The probe absorption can be controlled due to the Fano interference between the two decay paths. Such interference is similar to the decay - induced coherence in atomic systems with two closely lying energy states and occurs due to quantum interference in the electronic continuum [35].

The intensity of the Fano interference [35,41], defined by $p=\eta / \sqrt{\gamma_{2} \gamma_{3}}$, and the values $p=0$ and $p=1$ correspond to no interference (there is no negligible coupling between levels $|2\rangle$ and $|3\rangle$ ). This means that Fano interference does not generate) and perfect interference (no dephasing decay rates $\gamma_{i j}^{d p h}=0$ ), respectively. It is worth noting that the above described parameter $p$ is mainly controlled via the population decay rates $\gamma_{i}\left(\gamma_{1}, \gamma_{2}\right)$ and dephasing decay rates $\gamma_{i j}^{d p h}$, and it appears when the cross coupling term is present $(\eta \neq 0)$, but disappears when the cross coupling term is absent $(\eta=0)$. In order to study the effect of the quantum interference induced by incoherent pumping fields on the quantum well system, two broadband polarized fields $r_{1}$ and $r_{2}\left(r_{1}=r_{2}=r\right.$ )(can be provided by the diode laser that had a broad variable linewidth) that serve as the incoherent pumping fields and apply to the transitions $|1\rangle \rightarrow|2\rangle$ and $|1\rangle \rightarrow|3\rangle$, respectively (see Figs. 1(c) and 1(d)).

Thus, the Eq. 2 becomes as:

$$
\begin{array}{r}
d \rho_{12} / d t=-\frac{\gamma_{21}}{2} \rho_{12}+i\left(\Delta_{p}-1 / 2 \omega_{s}\right) \rho_{12}-r \rho_{12} \\
i \Omega_{p 2}\left(\rho_{22}-\rho_{11}\right)-i \Omega_{p 1} \rho_{32}-1 / 2(\eta+\eta) \rho_{13} \\
d \rho_{13} / d t=-\frac{\gamma_{31}}{2} \rho_{13}+i\left(\Delta_{p}+1 / 2 \omega_{s}\right) \rho_{13}-r \rho_{13} \\
i \Omega_{p 1}\left(\rho_{33}-\rho_{11}\right)-i \Omega_{p 2} \rho_{23}-1 / 2(\eta+\eta) \rho_{12}, \\
d \rho_{23} / d t=-\frac{\gamma_{32}}{2} \rho_{23}-i \omega_{2} r h o_{23}-r \rho_{23}+i \Omega_{p 2} \rho_{13} \\
-i \Omega_{p 1} \rho_{12}-1 / 2(\eta+\eta)(\rho 33+\rho 22)+\eta \rho_{11}, \\
d \rho_{22} / d t=-\gamma_{2} \rho_{22}+i \Omega_{p 2}\left(\rho_{12}-\rho_{21}\right) \\
-1 / 2(\eta+\eta)\left(\rho_{32}+\rho_{23}\right)+r\left(\rho_{11}-\rho_{33}\right) \\
d \rho_{33} / d t=-\gamma_{3} \rho_{33}+i \Omega_{p 1}\left(\rho_{13}-\rho_{31}\right) \\
-1 / 2(\eta+\eta)\left(\rho_{32}+\rho_{23}\right)+r\left(\rho_{11}-\rho_{33}\right) \\
\rho_{11}+\rho_{22}+\rho_{33}=1
\end{array}
$$

The incoherent fields are added accordingly to the method described in Refs.[42,43,44,45,46]. The quantum interference induced by incoherent pumping fields is described by a parameter $\eta=\sqrt{r_{1} r_{2}}$, which is
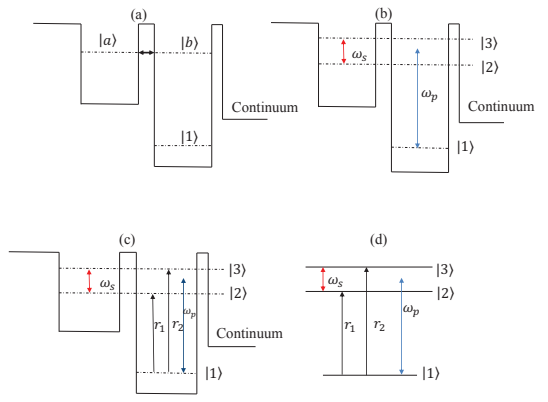

Fig. 1: (a) Energy level diagram of a double quantum well structure. It consists of two quantum wells and a collector region separated by thin tunnelling barriers. Subband $|a\rangle$ of the shallow well is resonant with the second subband $|b\rangle$ of the deep well. (b) Due to the strong coherent coupling via the thin barrier, the levels split into a doublet $|2\rangle$ and $|3\rangle$, which are coupled to a continuum by a thin tunnelling barrier adjacent to the deep well. $\omega_{s}$ denotes the splitting between the two upper levels (given by the coherent coupling strength) and $\omega_{p}$ is the weak probe laser.

added following the Ref. [44]. In fact, if the polarizations of the two incoherent fields $\varepsilon_{1}$ and $\varepsilon_{2}$ properly be arranged in such a way that $\varepsilon_{1} \cdot \mu_{21}=\varepsilon_{2} \cdot \mu_{31}=0$, thus one field acts on only one transition so that $\dot{\eta}=0$ which means there is no interference due to incoherent pump fields. However, when $\varepsilon_{1} . \mu_{21} \neq 0$ and $\varepsilon_{2} . \mu_{31} \neq 0$, one polarized broadband field can couple with more than one transition, and thus $\dot{\eta} \neq 0$.

The incoherent pumping fields have been studied experimentally [42] and theoretically $[43,44,45,46,52]$ in quantum coherent media, such as atomic medias [42,44], YAG crystal[45] and left-handedness materials[46]. It is worth noting that the SQW sample used in this paper is very much similar to the one reported in reference [35] thus, we can keep the same parametric conditions here, and the three-level system of electronic subbands can be grown on the substrate. Thus, the experimental feasibility of the scheme is evident. In order to drive the linear and nonlinear susceptibility, we need to obtain the steady state solution of the density matrix equations. The density matrix elements can be expressed as $\rho_{i j}=\rho_{i j}^{0}+\rho_{i j}^{1}+\rho_{i j}^{2}+\rho_{i j}^{3} \ldots$ Using the fact that the probe field is much weaker than the coupling field, the zeroth order solution will be $\rho_{11}^{0}=1$ and the other elements are equal to zero. The linear [47] and nonlinear [48] susceptibility are given by:

$$
\begin{gathered}
\chi^{(1)}=\frac{2 N}{\varepsilon_{0} \hbar \Omega_{p}}\left(\mu_{21}^{2} \rho_{21}^{1}+\mu_{31}^{2} \rho_{31}^{1}\right) \\
\chi^{(3)}=\frac{2 N}{3 \varepsilon_{0} \hbar^{3} \Omega_{p}^{3}}\left(\mu_{31}^{4} \rho_{31}^{3}+\mu_{31}^{4} \rho_{31}^{3}\right)
\end{gathered}
$$


Substituting coherence terms $\rho_{21}$ and $\rho_{31}$ into Eq.(2) and (3) and assuming $\mu_{21}=\mu_{12}=\mu$, the linear and nonlinear susceptibility are obtained as:

$\chi^{(1)}=\frac{2 N \mu^{2}}{\varepsilon_{0} \hbar Z}\left[2 \Delta_{p}+i / 2\left(\gamma_{31}+\gamma_{21}\right)+2 r-i(\eta+\eta ́)\right]$

and

$$
\begin{array}{r}
\chi^{(3)}=\frac{2 N \mu^{4}}{\varepsilon_{0} \hbar^{3} \Omega_{p}^{2} Z}\left\{\left[\rho_{22}^{2}-\rho_{11}^{2}+\rho_{32}^{2}\right]\left[\frac{\gamma_{12}}{2}-i\left(\Delta_{p}-\omega_{s} / 2\right)+r-1 / 2(\eta+\eta)\right)\right. \\
\left.+\left[\rho_{33}^{2}-\rho_{11}^{2}+\rho_{23}^{2}\right]\left[\frac{\gamma_{31}}{2}-i\left(\Delta_{p}+\omega_{s} / 2\right)+r-1 / 2(\eta+\eta)\right]\right\}
\end{array}
$$

where

$$
\begin{array}{r}
Z=-\Delta_{p}^{2}-i \Delta_{p}\left(1 / 2\left(\gamma_{21}+\gamma_{31}\right)+2 r\right)+r^{2} \\
r / 2\left(\gamma_{21}+\gamma_{31}\right)+\frac{i \omega_{s}}{4}\left(\gamma_{31}-\gamma_{21}\right)+\frac{\omega_{s}^{2}}{4}-\frac{(\eta+\eta)^{2}}{4}
\end{array}
$$

In this expressions we set $\Omega_{p 1}=\Omega_{p 2}=\Omega_{p}$, where $\Omega_{p}$ is to be real.

For further discussion in linear optical properties of the medium, we introduce the group index $n_{g}=\frac{c}{v_{g}}$, where $c$ is the speed of light in vacuum and the group velocity is given by $[49,50,51]$ :

$$
v_{g}=\frac{c}{1+2 \pi \operatorname{Re}\left(\chi\left(v_{g}\right)\right)+2 \pi v_{g} \frac{\partial}{\partial v_{g}} \operatorname{Re}\left(\chi\left(v_{g}\right)\right)}
$$

where $v_{g}$ is the frequency of the probe field .The linear dispersion and absorption are proportional to real and imaginary part of $\chi_{(1)}$, while the real and imaginary part of $\chi_{(3)}$ determine the nonlinear dispersion i.e. Kerr nonlinearity and the nonlinear absorption.

\section{Results and discussion}

Now, we intend to study the linear and nonlinear behavior of the three level semiconductor double QW structure. In the following numerical calculations, we assume that all subbands have the same effective mass and the electron-electron effects have very small influence on our results. Note that, in the following numerical calculations the choices of the parameters are based on experimental results from reference [35]. We will show that the electronic structure can be used as an optical medium to achieve a large Kerr nonlinearity with negligible probe absorption. This may be important for achieving large nonlinearities under low power conditions. Firstly, we are interested in the effect of the strength of Fano interference on linear and nonlinear susceptibility. The linear and nonlinear susceptibility of weak probe field is shown in Fig. $2(\mathrm{a}, \mathrm{b})$. it can be seen that for $\omega_{s}=17.6 \mathrm{meV}$ and when $p=0.46$, a weak Kerr nonlinearity is accompanied by strong linear and nonlinear absorption around zero probe field detuning. This situation is not desirable for application of low intensity nonlinear optics due to the thermal effects of devices. In order to eliminate the thermal effects, we propose conditions to reach an enhanced Kerr nonlinearity with reduced absorption in the medium just under the condition of slow light levels. (a)

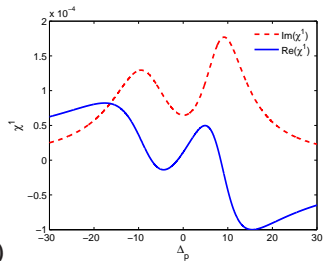

(c)

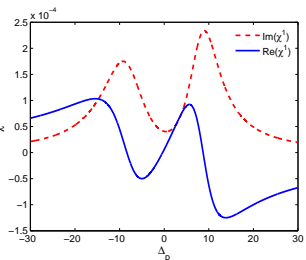

(e)

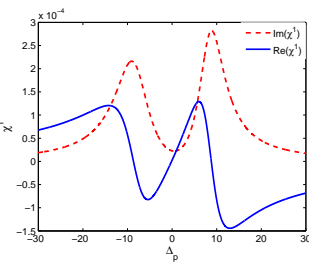

(b)

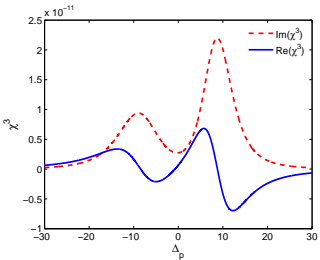

(d)

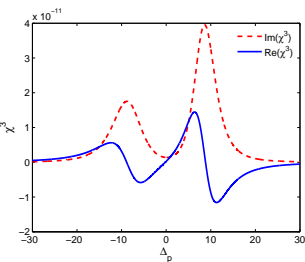

(f)

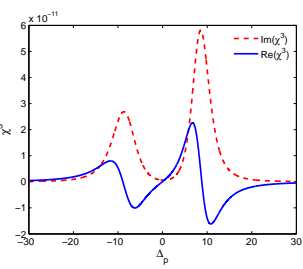

Fig. 2: Linear and nonlinear susceptibility versus probe field detuning. (a, c, e) Linear absorption (dashed line) and linear dispersion (solid line). (b, d, f) Nonlinear absorption (dashed line) and Kerr nonlinearity (solid line). The selected parameters are $(p=0.46): \gamma_{21}^{d p h}=$ $6 m e V, \gamma_{31}^{d p h}=9.2 m e V, \gamma_{32}^{d p h}=7.6 m e V(a, b) ;(p=0.63):$ $\gamma_{21}^{d p h}=3 \mathrm{meV}, \gamma_{31}^{d p h}=4.6 \mathrm{meV}, \gamma_{32}^{d p h}=4.8 \mathrm{meV}(\mathrm{c}, \mathrm{d}) ;(p=$ $0.77): \gamma_{21}^{d p h}=1.5 \mathrm{meV}, \gamma_{31}^{d p h}=2.3 \mathrm{meV}, \gamma_{32}^{d p h}=1.9 \mathrm{meV}$ (e, f). The other parameters are $\omega_{s}=17.6 \mathrm{meV}, \gamma_{2}=$ $5.6 \mathrm{meV}, \gamma_{3}=7 \mathrm{meV}, r=0, \eta=0$, and $\Omega_{p}=0.001 \mathrm{meV}$.

Investigation on Figs. 2 (c, d) and Fig. 2 (e, f) show that by increasing the strength of Fano interference to $p=0.63$ and even to $p=0.77$, the linear and nonlinear absorption decrease with respect to Fig. 2 (a, b), and the Kerr nonlinearity enhances around $\Delta_{p}=0$. Obviously, the more increasing the $p$ parameter, the more enhancement of the maximal Kerr nonlinearity, and simultaneousely, the more reduction of probe absorption around zero probe detuning. This may suggests a giant Kerr nonlinearity with reduced absorption only by the quality of Fano interference. Note that the slope of linear dispersion is positive in Fig. 2 which corresponds to subluminal light propagation.

Physically, the quantum interference parameter $\eta$ remains fixed when choosing $\gamma_{2}=5.6 \mathrm{meV}$ and 
$\gamma_{3}=7 \mathrm{meV}$, but the varying dephasing rates $\gamma_{i j}^{d p h}$ influence the strength of the Fano interference $p$. This can affects the linear and nonlinear susceptibilities. In order to gain deeper insight into the above problem, we refer the readers to Ref. [34], when the authors investigated the optical bi(multi)stable behavior of such electronic medium. Li et al. observed the reduction of the bistable threshold as they go from $p=0.46$ to $p=0.77$. They pointed out that the OB comes essentially from the Kerr nonlinearity. From a physical point of view, when increasing the $p$ parameter, the probe absorption can be reduced and at the same time, the Kerr nonlinearity of the medium enhances, which makes the cavity field easier to reach saturation. This may be useful to control the threshold value and the hysteresis cycle width of bistable curve. Thus, we show that our results are in a good agreement with results presented by $\mathrm{Li}$ et al. (a)
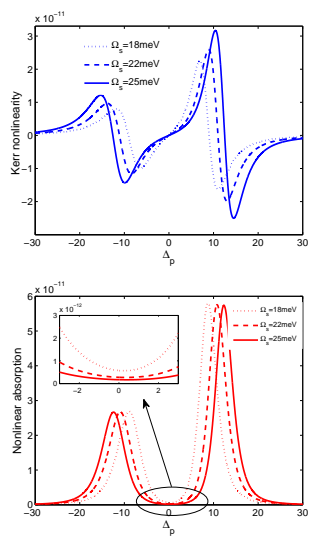

(b)

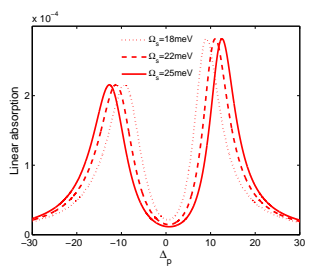

(a)

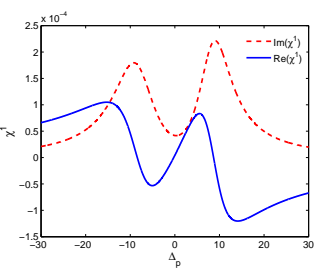

(c)

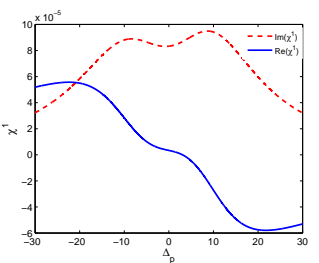

(b)

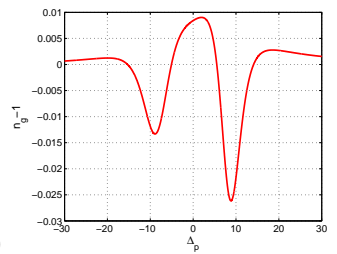

(d)

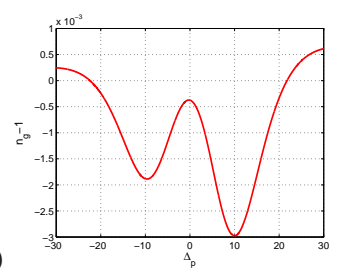

Fig. 4: Real and imaginary parts of the linear susceptibility $(a, c)$, and group index $(b, d)$ versus probe field detuning for the different rates of incoherent pumping fields. The parameters are $p=0.77$ and $(\mathrm{a}, \mathrm{b}), r=1 \mathrm{meV}(\mathrm{c}, \mathrm{d}), r=$ $9 \mathrm{meV}$. The other parameters are the same as Fig. 2.

Now, we will analyze how the incoherent pumping field affect on the probe field absorption, dispersion, and group index in Fig. 4. In the absence of quantum interference, i.e. $\eta=0$ and for given parameters, the slope of linear dispersion is definitely positive that is accompanied by an EIT dip (see Fig. 4( a)). Investigation on Fig. 4(c) shows that when the rates of incoherent pump field changes from $1=1 \mathrm{meV}$ to $1=9 \mathrm{meV}$, the slope of linear dispersion switchs from positive to negative that is accompanied by an absorption. We note that for a weak incoherent pumping rate $1=1 \mathrm{meV}$ the results shows that the subluminal light propagation occurs (Fig. 4(b)), while for strong incoherent pumping rates $(1=9 \mathrm{meV})$ the light propagation becomes superluminal (Fig. 4(d)). Physically, the coherence between levels $|2\rangle$ and $|3\rangle$ will be destroyed by the strong incoherent pumping from lower level $|1\rangle$ to upper levels $|2\rangle$ and $|3\rangle$. consequently, population in both upper levels cannot be trapped leading to enhancement of probe absorption.

To show the influence of the rate of incoherent pump $r$ on nonlinear behavior of the QW structure we give the plots of the real and imaginary parts of nonlinear susceptibility i.e. Kerr nonlinearity as well as nonlinear absorption as a function of $r$ for different strength of the barrier, the absorption for the probe field on the intersubband transitions $|1\rangle \rightarrow|3\rangle$ and $|1\rangle \rightarrow|2\rangle$ of the 
(a)


(b)

Fig. 5: Kerr nonlinearity (a) and nonlinear absorption (b) versus $r$ for different strength of Fano interference $p$. Reddot line $(p=0.46)$, black- dashed line $(p=0.63)$, and blue- solid line $(p=0.77)$. Delt $a_{p}=0.1 \mathrm{meV}$ and the other parameters are the same as Fig. 2.

Fano interference $p$ in Fig. 5. It is obvious from Fig. 5 (a) and Fig. 5(b) that increasing the rate of incoherent pump $r$ leads to a significant decrease of the kerr nonlinearity and nonlinear absorption. In addition, it can be seen that the dependence of the nonlinear dispersion and absorption on the strength of the Fano interference $p$ becomes very small for $r \geq 10 \mathrm{meV}$. According to this discussion, it can be realized that the incoherent pumping fields play a critical role to manipulate the Kerr nonlinearity behavior of the medium, as well as nonlinear absorption spectra. Thus, it can be used to reduce the effect of the strength of Fano interference $p$ on the Kerr nonlinearity. It is a key point that when the rate of incoherent pump reaches to a reasonable value, the effect of strength of Fano interference $p$ on Kerr nonlinearity of the electronic medium can be ignored, which suggests that we do not need to consider the effects of dephasing decay rates $\gamma_{i j}^{d p h}$ on nonlinear optical response of the medium. We can also ignore the processes such as electron-electron, interface roughness, and photon scattering process, because of their dependence on dephasing decay rates $\gamma_{i j}^{d p h}$, which make our scheme much more convenient in experimental realization. Note that these results can not be seen when the incoherent pumping fields are not considered. (a)

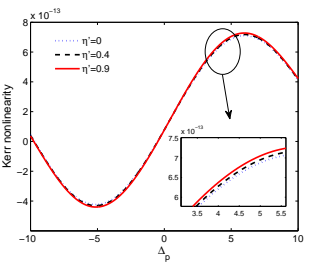

(b)

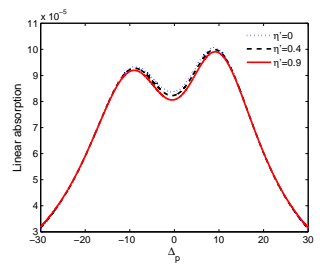

(c)

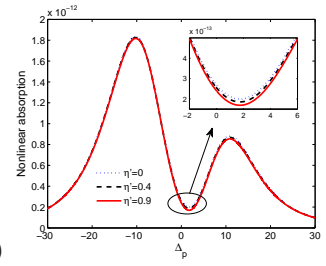

Fig. 6: Kerr nonlinearity (a), linear absorption (b), and nonlinear absorption versus probe field detuning for different values of $\eta=0 . r=8 \mathrm{meV}, p=0.77$ the other parameters are the same as Fig. 2.

Finally, we are interested in the the effect of the quantum interference arising from incoherent pumping fields $\dot{\eta}$ on Kerr nonlinearity behavior of the QW structure. The Kerr nonlinearity as a function of probe field detuning is displayed in Fig. 6(a). It is obvious that the Kerr nonlinearity is approximately insensitive to the quantum interference induced by incoherent pumping fields $\dot{\eta}$. We also display the linear and nonlinear absorption spectra in Figs. 6(b) and 6(c). It is shown that both the linear and nonlinear absorption of the electronic medium change weakly with the increasing quantum interference induced by incoherent pumping fields $\dot{\eta}$ which are verified by the above analysis. A reasonable explanation for this is that the quantum interference $\eta$ cannot suppress the absorption and thus, influence the nonlinearity of the electronic medium.

\section{Conclusions}

We have theoretically investigated the linear and nonlinear optical properties of a three subband asymmetric semiconductor quantum well system driven by a probe field. Some of the important results have been demonstrated in following. - An enhanced Kerr nonlinearity with reduced absorption can be achieved just via the strength of Fano type interference under the condition of slow light levels. - Superluminal light propagation can be switched to subluminal light propagation only by the rate of an incoherent pump field. - It is shown that the incoherent pumping fields play an important role to manipulate the Kerr nonlinearity behavior of the medium, so that it can be used to reduce the effect of the strength of Fano interference on the refractive part of third order susceptibility. - Kerr nonlinearity is insensitive to the quantum interference 
induced by incoherent pumping fields. It is worth noting that we could achieve the giant Kerr nonlinearity under the condition of slow light levels in the absence of any external coherent driving fields. In other words, this way is different from the conventional way in ordinary laser-driven schemes that coherent driving fields are necessary to enhance the Kerr nonlinearity. As a result, it may provide some new possibilities for technological applications in optoelectronics and solid-state quantum information science.

\section{Acknowledgement}

The authors would like to acknowledges Mr. Ali KhalediNasab for his technical support and contribution on this work.

\section{References}

[1] S. E. Harris, Electromagnetically induced transparency, Phys., 50, 36 (1997).

[2] A. G. Litvak, M. D. Tokman, Electromagnetically induced transparency in ensembles of classical oscillators, Phys. Rev. Lett., 88, (2002).

[3] S. E. Harris, J. E. Field, A. Imamoglu, Nonlinear optical processes using electromagnetically induced transparency, Phys. Rev. Lett., 64, (1990).

[4] E. Arimondo, in: E. Wolf (Ed.), Progress in Optics, XXXV, North-Holland, Amsterdam, 257 (1996).

[5] H. R. Xia, C. Y. Ye, S. Y. Zhu, Experimental observation of spontaneous emission cancellation, Phys. Rev. Lett., 77, 1032 (1996).

[6] M. Mahmoudi, M. Sahrai, H. Tajalli, Phys. Lett., A 357, 6671 (2006).

[7] H. Tajalli, M. Sahrai. J. Opt. B: Quantum Semiclass.Opt., 7, 168- 173 (2005).

[8] J. Mompart, R. Corbalan, Lasing without inversion, J. Opt. B: Quantum Semiclassical Opt., 2, R7 (2000).

[9] A. E. Allahverdyan, R.S. Gracia, T. M. Nieuwenhuizen, Work extraction in the spin-boson model, Phys. Rev., E 71, 046106 (2005).

[10] Yueping Niu, and Shangqing Gong, Phys. Rev., A 73, 053811 (2006).

[11] X. Yan, L. Wang, B. Yin, W. Jiang, H. Zheng, J. Song, Y. Zhang, Phys. Lett., A 372, 6456 (2008).

[12] Y. P. Niu, S. Q. Gong, R. X. Li, Z. Z. Xu, X. Y. Liang, Opt. Lett., 30, (2005).

[13] Hamid Reza Hamedi, Seyyed Hossein Asadpour, Mostafa Sahrai, Optik, 124, 366- 370 (2013).

[14] Eyob A. Sete and Hichem Eleuch, Phys. Rev., A 85, 043824 (2012).

[15] A. Baas, J. Ph. Karr, H. Eleuch, and E. Giacobino, Phys. Rev., 69, 023809 (2004).

[16] H. R. Hamedi, Ali Sari, M. Sahrai, and S.H. Asadpour, Commun. Theor. Phys., 59, 199-204 (2013).

[17] Zhen Wang,1 Ai-Xi Chen,2 Yanfeng Bai,1 Wen-Xing Yang, 1,3,* and Ray-Kuang Lee, J. Opt. Soc. Am., B 29, 2891 (2012).
[18] M. M. Kash, V. A. Sautenkov, A. S. Zibrov, L. Hollberg, G. R. Welch, M. D. Lukin, Y. Rostovtsev, F. S. Fry, M. O. Scully,Phys. Rev. Lett., 82, 5229 (1999).

[19] S. H. Asadpour, Hamid Reza Hamedi, A. Eslami-Majd, M. Sahrai. Physica E, 44, 464 (2011).

[20] Y. F. Xiao, K. Sahin, V. Gaddam, C. Hau, N. Imoto, Lan Yang, Opt. Express, 16, 21462 (2008).

[21] J. Howell, J. Yeazell, Phys. Rev., A 62, 032311 (2000).

[22] K. Mattle, H. Weinfurter, P. G. Kwiat, A. Zeilinger, Phys. Rev. Lett., 76, 4656 (1996).

[23] G. D'Ariano, L. Maccone, M. G. A. Paris, M. F. Sacchi, Phys. Rev., A 61, 053817 (2000).

[24] A. Joshi, A. Brown, H. Wang, and M. Xiao, Phys. Rev., A 67, 041801(R) (2003).

[25] S. Singh, J. Rai, C. M. Bowden, and A. Postan, Phys. Rev., A 45, 5160-5165 (1992).

[26] P. Galatola, L. A. Lugiato, M. G. Porreca, and P. Tombesi, Opt. Commun., 81, 175-178 (1991).

[27] Gediminas Jonusauskas, Roaldas Gadonas, Claude Rullitxe. Optics Communications, 112, 80-84 (1994).

[28] Yanfeng Bai, Wenxing Yang, Xiaoqiang Yu. Optics Communications, 283, 5062-5066 (2010).

[29] S. E. Harris, Y. Yamamoto. Photon switching by quantum interference. Phys. Rev. Lett., 81, 3611-3614 (1998).

[30] M. Yan, E. G. Ricky, Y. Zhu. Phys. Rev., A 64, 041801 (2001).

[31] M. Sahrai, H. R. Hamedi, M. Memarzadeh. Journal of Modern Optics, 59, 980-987 (2012).

[32] Zhiping Wang, Hongyi Fan. Journal of Luminescence, 130, 2084-2088 (2010)

[33] Wen-Xing Yang, Ting-Ting Zha, Ray-Kuang Lee. Physics Letters, A 374, 355-359 (2009).

[34] J. H. Li and X. X. Yang. Eur. Phys. J., B 53, 449-454 (2006).

[35] A. Imamog?lu, R.J. Ram, Opt. Lett., 19, 1744 (1994); H. Schmidt, K. L. Campman, A. C. Gossard, A. Imamog?lu, Appl. Phys. Lett., 70, 3455 (1997).

[36] J. Faist, F. Capasso, C. Sirtori, K. West, L.N. Pfeiffer, Nature, 390, 589 (1997); M. D. Frogley, J.F. Dynes, M. Beck, J. Faist, C.C. Phillips, Nature Mater., 5, 175 (2006).

[37] A. Joshi, Phys. Rev., B 79, 115315 (2009).

[38] A. Joshi, M. Xiao, Appl. Phys., B 79, 65 (2004); J. H. Li, X. Yang, Eur. Phys. J., B 53, 449 (2006); J. H. Li, Phys. Rev., B 75, 155329 (2007).

[39] Z. Wang, Opt. Commun., 282, 4745 (2009); K. I. Osman, S. S. Hassan, A. Joshi, Eur. Phys. J. D, 54, 119 (2009).

[40] G. B. Serapiglia, E. Paspalakis, C. Sirtori, K. L. Vodopyanov, C. C. Phillips, Phys. Rev. Lett., 84, 1019 (2000); J. F. Dynes, M. D. Frogley, M. Beck, J. Faist, C. C. Phillips, Phys. Rev. Lett., 94, 157403 (2005).

[41] J. H. Wu, J. Y. Gao, J. H. Xu, L. Silvestri, M. Artoni, G.C. La Rocca, F. Bassani, Phys. Rev. Lett., 95, 057401 (2005).

[42] M. D. Lukin and A. Imamo?glu, Phys. Rev. Lett., 84, 1419 (2000).

[43] Y. Zhu, Phys. Rev., A 55, 4568 (1997).

[44] W. H. Xu, J. H. Wu and J. Y. Gao, J. Phys., B 39, 1461 (2006).

[45] H. F. Zhang, J. H. Wu, X. M. Su and J. Y. Gao, Phys. Rev., A 66, 053816 (2002).

[46] X. M. Su, H. X. Kang, J. Kou, X. Z. Guo and J. Y. Gao, Phys. Rev., A 80, 023805 (2009). 
[47] M. O. Scully, M. S. Zubairy, Quantum Optics, Cambridge University Press, Cambridge, UK, (1997).

[48] R. W. Boyd, Nonlinear Optics, Academics, San Diego, (1992).

[49] L. J. Wang, A. Kuzmich, A. Dogariu, Nature, 406, 277 (2000).

[50] O. Kocharovskaya, Y. Rostovtsev, M. O. Scully, Phys. Rev. Lett., 86, 628 (2001).

[51] C. Liu, Z. Dutton, C.H. Behroozi, L.V. Hau, Nature, 409, 490 (2001).

[52] Victor V. Kozlov, Yuri Rostovtsev, and Marlan O. Scully. Phys. Rev., A 74, 063829 (2006). 